\title{
Investigator Status in Study
}

National Cancer Institute

\section{Source}

National Cancer Institute. Investigator Status in Study. NCI Thesaurus. Code C70725.

A category used to describe the registration status of a clinical study investigator in a particular study. 